\title{
Redundancia en Redes de Comunicación para la Automatización y Protección de Sistemas de Potencia Eléctrica con IEC 61850
}

\author{
Juan C. Carreño-Pérez ${ }^{1}$, Juan P. Morales-Rivera' ${ }^{1}$ y Edwin Rivas-Trujillo ${ }^{*}$ \\ (1) Grupo Energía Bogotá, Gerencia de Mantenimiento, Bogotá - Colombia. \\ (e-mail: juan_carlos_carreno@hotmail.com, juanp1116@hotmail.com) \\ (2) Facultad de Ingeniería, Proyecto Curricular Ingeniería Eléctrica, Universidad Distrital Francisco José de \\ Caldas, Bogotá - Colombia. (e-mail: erivas@udistrital.edu.co)
}

* Autor a quien debe ser enviada la correspondencia

Recibido May. 4, 2018; Aceptado Jul. 9, 2018; Versión final Ago. 20, 2018, Publicado Feb. 2019

\begin{abstract}
Resumen
En este artículo se analizan de manera detallada los protocolos de redundancia utilizados para redes de automatización y protección de sistemas de potencia eléctrica. Con base en los requerimientos para las redes de comunicaciones, servicios de protocolos y servicios de mensajería presentados en la norma IEC 61850; se hace necesario el uso de protocolos de red que garanticen tiempos máximos de falla a nivel de comunicación. Como solución, la edición 2 de la norma IEC 61850, referencia la utilización de dos de los protocolos de redundancia expuestos en la norma IEC 62439-3 PRP-HSR, los cuales son el objeto de estudio del presente artículo. Adicionalmente, se realizó el diseño y la implementación para el caso de estudio de una subestación eléctrica de $230 \mathrm{kV}$ del Grupo Energía Bogotá, con un este esquema de redundancia hibrido HSRPRP. Se validó y analizó su comportamiento obteniendo resultados satisfactorios para los servicios requeridos por el sistema de control y protección de la Subestación.
\end{abstract}

Palabras clave: IEC 61850; IEC 62439; IED; HSR; Iatencia; PRP; RedBox; conmutación

\section{Redundancy in Communication Networks for the Automation and Protection of Electrical Power Systems with IEC 61850}

\begin{abstract}
This article describes a detailed study and analysis of the redundancy protocols used for automation networks and power systems. According to requirements for communications network, protocol services and messaging services, presented in the IEC 61850 standard, the use of network protocols is required to ensure the maximum time allowed in communications failure. As a solution, the edition 2 of IEC 61850 standard, refers to use of two redundancy protocols according to the IEC 62439-3 PRP- HSR standard, which are part of the study object. Also, the design and implementation for the study case of a 230kV electrical substation of the Group Energía Bogotá was carried out, with a hybrid redundancy scheme HSR-PRP. Its behavior was analyzed and validated obtaining satisfactory results for the services required by the control and protection system of the Substation.
\end{abstract}

Keywords: IEC 61850; IEC 62439; IED; HSR; latency; PRP; RedBox; switch 


\section{INTRODUCCIÓN}

La tendencia en el diseño e implementación de los sistemas de control y protección de las subestaciones eléctricas de alta tensión, es automatizar con el fin de mitigar errores y garantizar los tiempos de respuesta requeridos por el sistema de potencia para su correcto funcionamiento. A su vez, esta automatización adquiere mayor importancia a medida que los enfoques muestran inclinaciones hacia sistemas inteligentes, integrados y distribuidos (Zapata, 2011). La norma IEC 61850 es un estándar para la automatización de subestaciones eléctricas, dentro de los aspectos más relevantes se encuentran la interoperabilidad del protocolo de comunicación y la transmisión de información en tiempo real del protocolo IEC 61850, que se realiza a través servicios de comunicación como la transferencia rápida de eventos GOOSE (Generic Object Oriented Substation Event), mensajes MMS (Manufacturing Message Specification) y los Sampled Values (Goraj, 2016). La utilización de estas características de transmisión de datos permite reducción considerable del cableado de cobre convencional de la instalación eléctrica. Para cumplir con los requerimientos de tiempos de respuesta, el estándar IEC 61850 en su capítulo cinco detalla los tiempos máximos de conmutación y latencia de la red de comunicaciones a partir de la funcionalidad que se vaya a implementar (Kumar et al., 2016).

Dentro de la norma no se define ninguna topología de red en particular, sin embargo en su segunda edición referencia la necesidad de utilizar algunos mecanismos para diseñar una red robusta para contrarrestar fallas y en lo posible no tener tiempos de conmutación. Aunque la norma no descarta el uso de topologías redundantes a través de protocolos de capa 2 muy utilizados en redes comunes de comunicación, como STP (IEEE std. 802.1D, 1990), RSTP (IEEE std. 802.1D, 2004) y enlaces duales radiales, contemplando tiempos de convergencia de red ante fallas que pueden estar en el orden de los 100ms (Goraj, 2016). Se propone reducir los tiempos de conmutación y aumentar la probabilidad de determinismo en la red, para lo cual se recomienda el uso de la norma IEC 62439-3 Clausulas 4 y 5 que hacen énfasis en los protocolos PRP y HSR respectivamente (IEC 62439-3, 2012).

En el presente artículo se realiza un caso de estudio para una Subestación Eléctrica del Grupo Energía Bogotá ubicada dentro del anillo de transmisión de $230 \mathrm{kV}$ de la ciudad de Bogotá-Colombia, en la que se implementaron los protocolos PRP Parallel Redundancy Protocol (PRP) enunciada en la IEC62439-3:2012 cláusula 4, el cual se basa en el funcionamiento en paralelo de dos redes de área local y el protocolo High Availability Seamless Ring (HSR), que es propuesto en la norma IEC62439-3:2012 cláusula 5 y aplica el principio de PRP pero a diferencia de este, HSR utiliza una red común en configuración anillo y con estructura paralela (Jiang, 2009). Para el caso de estudio se realizaron pruebas funcionales y se sometió la red de comunicaciones a pruebas de robustez, avalancha de información y saturación de la red con el fin para analizar su comportamiento. Se pretendía indagar sobre los tiempos de conmutación ante fallas y pérdidas de información de los sistemas de control y protecciones, analizar la interoperabilidad y estabilidad a nivel de red y finalmente validar el correcto funcionamiento de los protocolos de redundancia de red y que estos cumplan con los requerimientos indicados en la norma IEC61850, para la mensajería GOOSE y bus de proceso.

\section{ANTECEDENTES Y ESTADO ACTUAL}

Los tiempos de recuperación máximo requerido ante una falla de comunicación entre los diferentes agentes participantes de una red, de acuerdo a la norma IEC 61850-5 se presenta en la Tabla 1 (Kirrmann et al., 2009). Esta información permite tener un punto de referencia para analizar los protocolos de redundancia que se pretendan implementar en un sistema, dependiendo del tipo de comunicación que se requiera establecer.

Tabla 1: Tiempos de conmutación requeridos en los servicios IEC 61850. Tol=Tolerancia de recuperación de la aplicación; t=Tiempo requerido para la recuperación de la comunicación

\begin{tabular}{|l|l|l|l|}
\hline $\begin{array}{l}\text { Comunicaciones entre participantes } \\
\text { de una red IEC 61650 }\end{array}$ & Servicio & Tol & $T$ \\
\hline SCADA a IED ->Cliente-Servidor & IEC 61850-8-1 & $800 \mathrm{~ms}$ & $400 \mathrm{~ms}$ \\
\hline IED a IED ->Enclavamientos & IEC 61850-8-1 & $12 \mathrm{~ms}$, (con Tminconf en 4ms) & $4 \mathrm{~ms}$ \\
\hline IED a IED ->Revertir Bloqueo & IEC 61850-8-1 & $12 \mathrm{~ms}$, (con Tminconf en 4ms) & $4 \mathrm{~ms}$ \\
\hline $\begin{array}{l}\text { Disparo de Protección ->excluyendo } \\
\text { la diferencial de barras }\end{array}$ & IEC 61850-8-1 & $8 \mathrm{~ms}$ & $4 \mathrm{~ms}$ \\
\hline Protección diferencial de barras & $\begin{array}{l}\text { IEC 61850-9-2 } \\
\text { en el bus de estación }\end{array}$ & $<1 \mathrm{~ms}$ & $\begin{array}{l}\text { Conmutación } \\
\text { instantánea }\end{array}$ \\
\hline Sampled Values & $\begin{array}{l}\text { IEC 61850-9-2 } \\
\text { en el bus de proceso }\end{array}$ & $\begin{array}{l}\text { Menor a dos muestras } \\
\text { consecutivas }\end{array}$ & $\begin{array}{l}\text { Conmutación } \\
\text { instantánea }\end{array}$ \\
\hline
\end{tabular}


En la actualidad fabricantes como (SIEMENS con su familia de productos SIPROTEC 4 y 5 , Ruggedcom y Scalance., HIRSCHMANN con RedBox RBX, OMICRON con su CMC 850, SEL con su familia de relés de protección, GE Energy con IED's de la familia UR y Micom, ABB con IED's Relion, Schneider Electric con el IED P142, MOXA Switch y RedBox.) tienen implementados los protocolos de red HSR o PRP presentados en la norma IEC 62439-3 en sus equipos, tanto en IED's como en dispositivos de red (UCAlug, 2012). En consecuencia a este desarrollo tecnológico que ofrece nuevos servicios de comunicación para los sistemas de automatización, surge la necesidad de activar mecanismos redundantes con el fin de reducir tiempos de restablecimiento ante una falla en la red (Molina et al., 2016).

El comité IEC SC65 WG15 "Highly Available Automation Networks", publica en abril del 2010 la norma IEC 62439 aplicable a redes de automatización de alta disponibilidad basado en el ISO / IEC 8802-3 (IEEE 802.3) (Araujo et al., 2013), con el fin de proporcionar métodos de redundancia a nivel de redes Ethernet. En el 2012 se realiza la publicación de una segunda edición de la IEC 62439-3:2012 la cual es referenciada en la norma IEC61850 Ed.2 "Communication Network and Systems for Power Utility Automation" en sus capítulos 5, 8-1 y 9-2, con el fin de brindar redundancia a nodos más complejos y redes más robustas como es el caso de las subestaciones y centrales eléctricas (IEC 61850-5, 2012). La norma sugiere la utilización de los protocolos descritos en la norma IEC 62439-3 Clausulas 4 y 5 que hacen énfasis en los protocolos PRP y HSR respectivamente.

\section{PRP (PARALLEL REDUNDANCY PROTOCOL)}

La estructura redundante de PRP se basa en tener redundancia en cada nodo denominado DANP (Doubly Attached Node with PRP). Este se une a dos redes de área local independientes, las cuales pueden tener diferentes topologías (Araujo et al., 2013). Cada nodo tiene dos puertos que operan en paralelo y que están conectadas a las mismas capas superiores de la pila de comunicación a través de la Entidad de Enlace de Redundancia LRE (Link Redundancy Entity) (Figura 1) ("IEC 61850-7-2 Communication networks and systems in substations -," 2003).

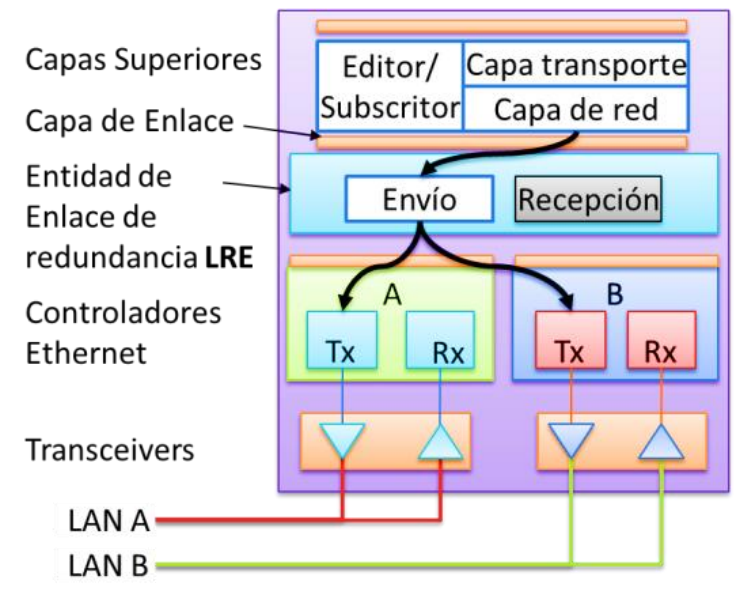

Fig. 1: LRE en el módulo de comunicación.

La base principal de PRP es el uso de dos redes física Ethernet independientes para la transmisión de datos, la trama es replicada por el nodo de envío y es trasmitida a ambas redes de forma simultánea, como se ilustra en la Figura 2. En el receptor es aceptada la primera trama que recibe y descartada la segunda, este proceso se realiza a través del LRE (IEC 62439-3, 2012).

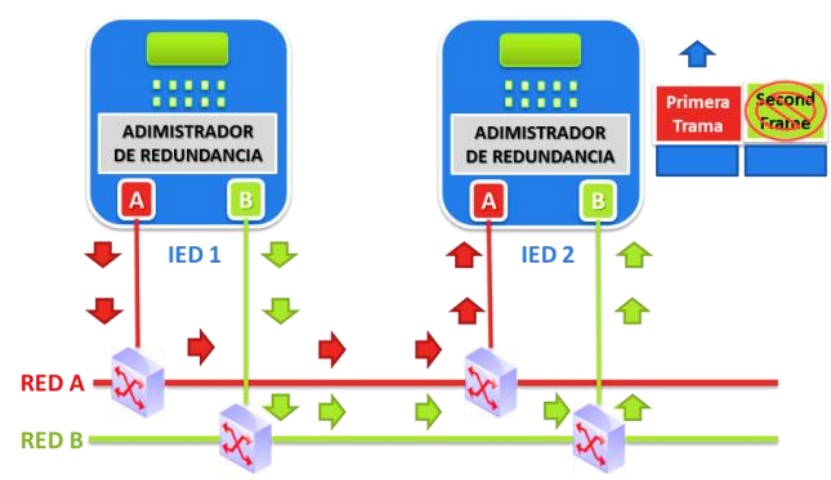

Fig. 2: Configuración PRP. 


\section{Manejo de duplicados}

Desde un DANP se recibe la misma trama sobre ambos adaptadores en funcionamiento, se debe mantener una trama e ignorar el duplicado (Figura 3). Es posible descartar la trama duplicada en el receptor gracias a la adición de seis octetos dentro de ella, esta información es denominada Redundancy Control Trailer (RCT) y es insertada por el LRE en cada trama recibida antes de enviarlos a la red (Araujo et al., 2013).

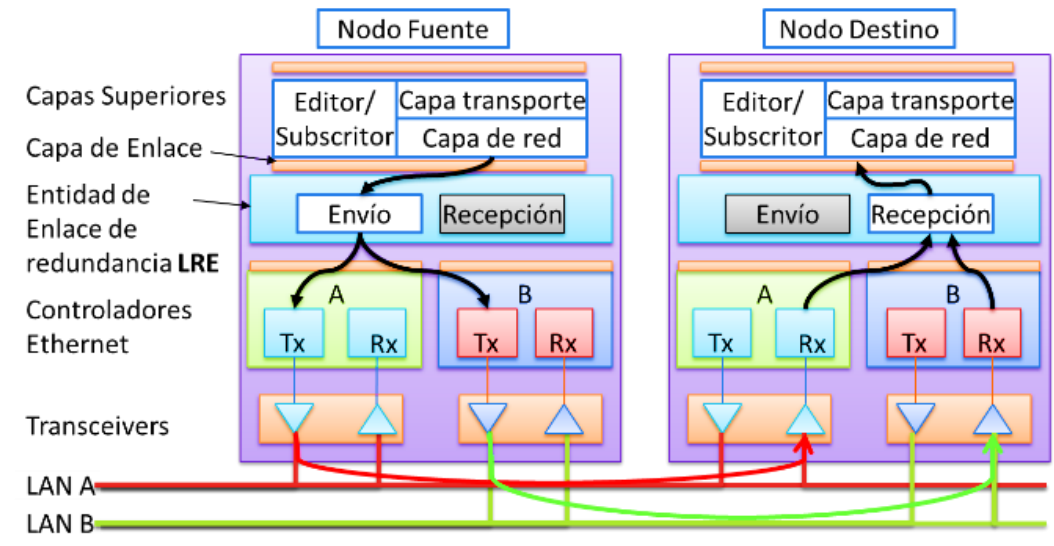

Fig. 3: Flujo de información en PRP.

\section{HSR (HIGH-AVAILABILITY SEAMLESS REDUNDANCY)}

HSR o Anillo homogéneo de alta disponibilidad, se propone basado en el protocolo PRP y permite una reducción significativa de los costes de hardware, ya que son necesarios menos switches y conexiones físicas, en lugar de esto se cuenta con un switch o conmutador en cada nodo final que ejecuta los algoritmos necesarios para la operación de HSR. Aunque la capa de red y los algoritmos de PRP y HSR son casi los mismos, en HSR no se puede aplicar todos los conceptos de PRP debido a su topología de anillo y al escenario de aplicación de HSR, que se caracteriza por el alto tráfico de la red (Araujo et al., 2012). HSR conserva los principios de PRP y su propiedad de tiempo cero de conmutación, aplicado a topologías en anillo y sub anillos (Figura 4). Con respecto al PRP, HSR permite reducir la infraestructura de red. Referente a los anillos basados en IEEE 802.1D (RSTP), IEC 62439-2 (MRP), IEC 62439-6 (DRP) o IEC 62439-7 (RRP), el ancho de banda disponible para el tráfico de red se reduce en algunos casos en función al tipo de tráfico (IEC 62439-3, 2012).

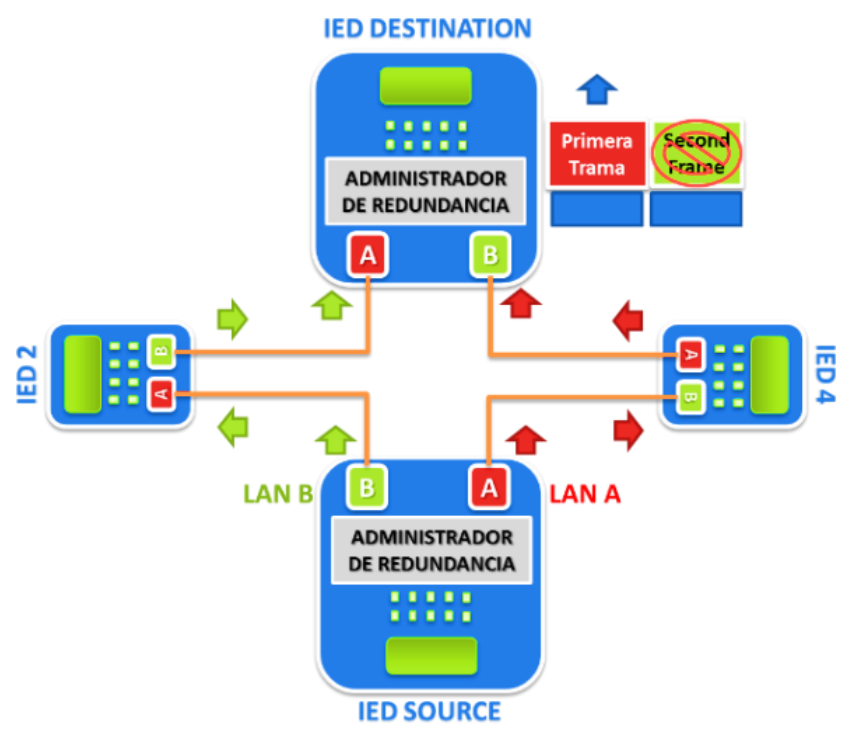

Fig. 4: Configuración HSR.

\section{Principio de operación}

Similar a PRP un nodo HSR tiene dos puertos operando paralelamente, el cual es denominado DANH (Doubly Attached Node with HSR protocol). Una red simple HSR consiste de nodos DANH, cada uno de ellos con dos puertos conectados en configuración anillo con enlaces full-duplex (Figura 5). El DANH fuente envía la trama que viene de las capas superiores de comunicación adicionando las etiquetas HSR para identificar las tramas duplicadas en los destinos. La trama es enviada simultáneamente sobre cada puerto (Trama A y Trama B). 


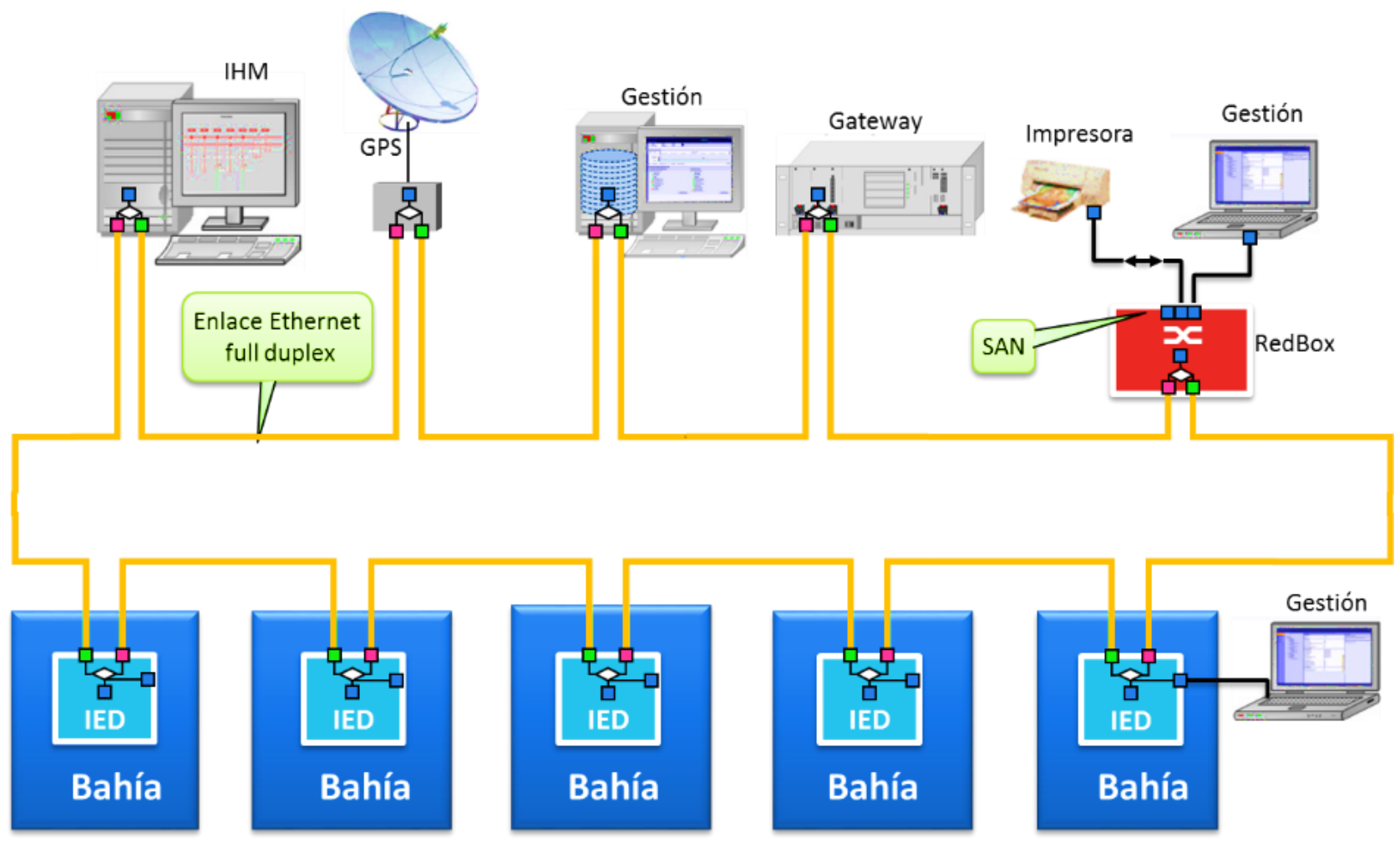

Fig. 5: Topología general HSR.

EI DANH destino recibe en condiciones normales, dos tramas iguales desde cada uno de sus puertos con cierto intervalo de tiempo, luego remueve las etiquetas HSR de la primera trama que recibe antes de enviarlo a las capas superiores de comunicación y descarta el duplicado. El tiempo de desviación entre las dos tramas depende de la posición relativa del nodo destino con respecto al nodo fuente. Los nodos simples (SAN), como servidores, impresoras, computadoras portátiles y otros equipos terminales, no se pueden conectar directamente al anillo HSR ya que la mayoría de ellos no son capaces de interpretar las etiquetas HSR de las secuencias. Previendo este fenómeno, la norma propone como solución un dispositivo de red adicional llamado RedBox (Redundancy Box).

\section{ARQUITECTURA IMPLEMENTADA}

Dadas las limitantes en cuanto al manejo de protocolos HSR y PRP de algunos equipos que hacen parte del sistema de control y protección en subestaciones eléctrica de alta tensión, como lo son Gateways, RTU, GPS, Switches, estaciones de trabajo, registradores de fallas, entre otros, y teniendo en cuenta que equipos descritos en la IEC 62439 no se habían desarrollado como es el caso del QUADBOX, no era posible implementar una solución completamente unificada con alguno de los protocolos de redundancia de red. Lo anterior nos llevó a un diseño de red integral que brindara una alta confiabilidad y el rendimiento requerido para el correcto funcionamiento del sistema.

Bajo los análisis realizados se construyeron varias propuestas con base en las tecnologías desarrolladas a la fecha por varios fabricantes y orientados a cumplir con los requerimientos necesarios para el sistema de control y protección de subestaciones eléctricas. Como resultado se definieron para el Grupo Energía Bogotá, dos arquitecturas las cuales se presentan en los siguientes párrafos.

La primera arquitectura diseñada hace referencia a una topología hibrida como se muestra en la Figura 6 , con anillos HSR entre los IED's y una conversión a una red PRP en la que se encuentran conectados los Gateway de la subestación, equipos de gestión y IHM's. Una de las características más importantes de esta configuración es que los anillos HSR no tienen más de 10 dispositivos, con lo que se garantiza que en el peor de los escenarios el tiempo de latencia es de aproximadamente $3 \mathrm{~ms}$, cumpliendo con los tiempos requeridos para el correcto funcionamiento de todos los servicios de la Norma IEC61850. 


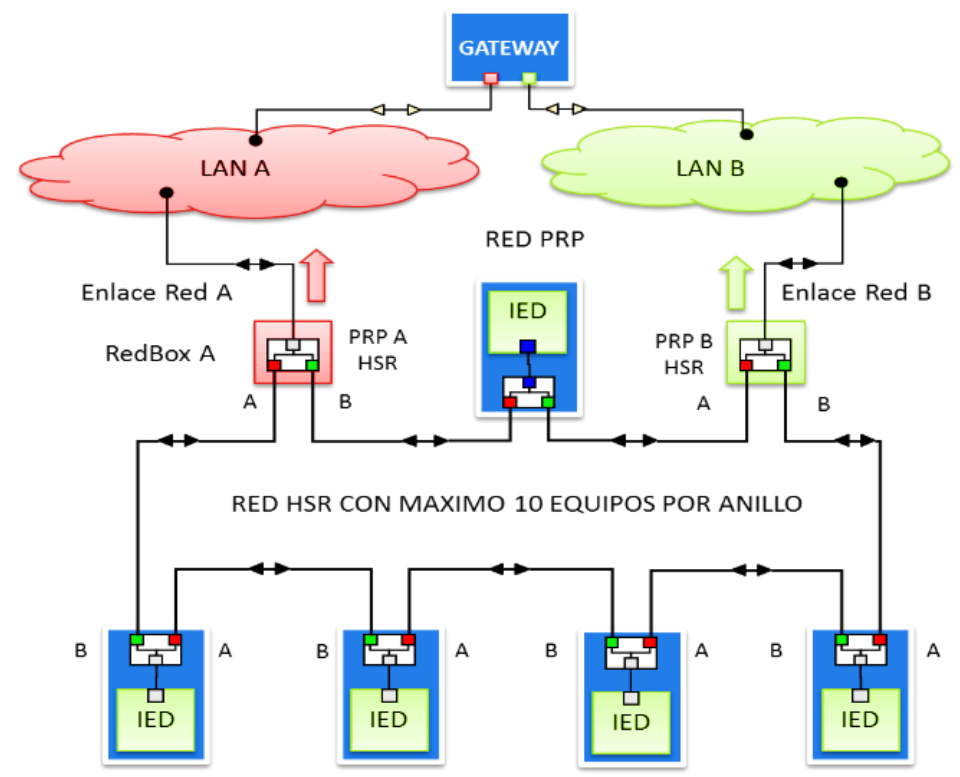

Fig. 6: Topología hibrida HSR a red PRP implementada.

La segunda topología de red implementada es un esquema de red PRP en la que los IED's, Gateway de la subestación, equipos de gestión y IHM's, son conectados a dos redes LAN, cada una con switches en topología anillo con protocolo de redundancia RSTP como se presenta en la Figura 7. Con esta infraestructura se garantiza un mayor nivel de confiabilidad a cada una de las redes PRP con lo que se logra mejorar la disponibilidad del sistema.

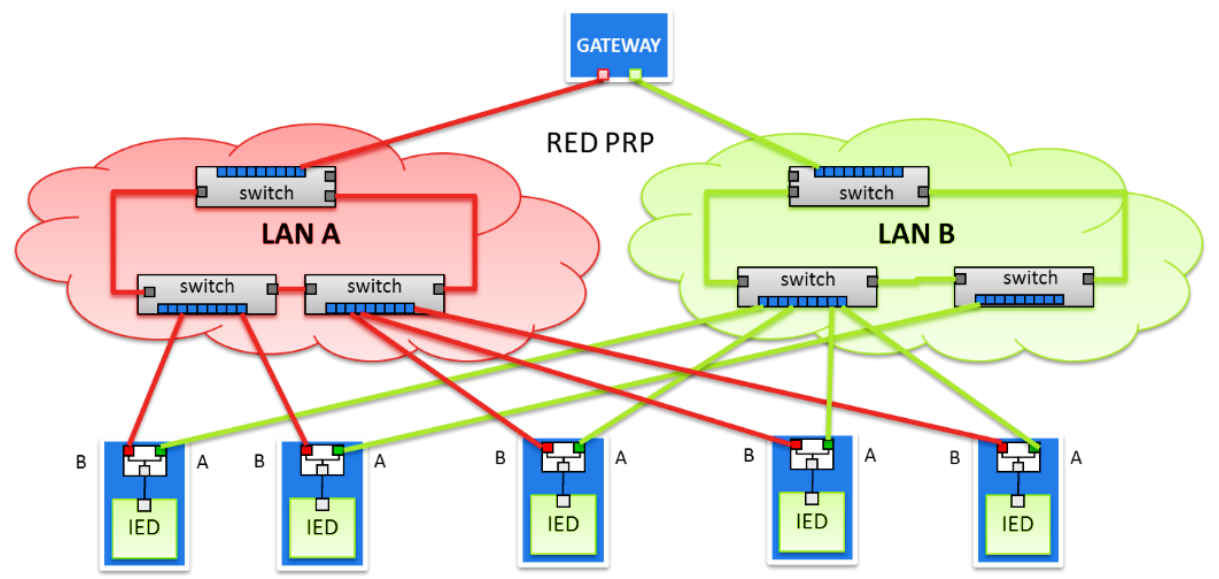

Fig. 7: Topología PRP implementada.

Sin embargo, a pesar de que las dos propuestas mejoran la confiabilidad, disponibilidad y seguridad del sistema, uno de los aspectos analizado fue la cantidad de dispositivos de red requeridos para la implementación de la solución, lo que incrementa los costos de la red, así como el número de equipos para gestionar y mantener durante el ciclo de vida de los activos. La Tabla 2 presenta las cantidades de equipos de red de comunicaciones requeridos según las topologías propuestas de arquitectura hibrida (HSR \& PRP) y PRP. Para la topología PRP hay un incremento de hardware comparado con la topología hibrida en un $45.7 \%$, lo que fue determinante al momento de selección la solución para una de las subestaciones de $230 \mathrm{kV}$ más importantes de la empresa.

Tabla 2: Número de elementos de red de comunicaciones topologías

\begin{tabular}{|l|c|c|}
\hline \multicolumn{1}{|c|}{ Hardware } & Topología Híbrida & Topología PRP \\
\hline \# Red Box HSR & 8 & 0 \\
\hline \# Red Box PRP & 8 & 8 \\
\hline \# enlaces & 119 & 176 \\
\hline \# Switches & 7 & 18 \\
\hline \# Puertos & 238 & 352 \\
\hline
\end{tabular}


Basado en lo anterior se escogió como arquitectura solución la topología hibrida, sin embargo, debíamos garantizar en que la latencia estuviera acorde a los tiempos requeridos por los servicios utilizados en el sistema. Con base en esto y asumiendo el peor de los casos en el que cada nodo en el anillo está transmitiendo al mismo tiempo y con tramas con el mayor tamaño posible de 1528 octetos, cada nodo introduce una latencia aproximada de $123 \mu \mathrm{s}$ en una red de 100Mbit/s. El peor escenario de respuesta se obtiene al suponer el tamaño máximo del paquete, donde el tiempo de transmisión del mensaje Tp en un tramo de red viene dado por la ecuación 1 (RuggedCom Inc. I 30 Whitmore Road, 2008).

$$
T_{P}=1528 \text { byte } \times 8 \frac{\text { bits }}{\text { byte }} \times \frac{1}{100 \mathrm{Mbps}}=122 \mu 2
$$

El tiempo de latencia del mensaje $\left(T_{m}\right)$ para pasar de un nodo a otro (de un IED a otro) está dado por ecuación 2 (RuggedCom Inc. I 30 Whitmore Road, 2008):

$$
T_{m}=\left(T_{P} \times C_{1}+T_{1}\right)+\left(T_{P} \times C_{2}+T_{1}\right)
$$

Dónde: $\mathrm{c} 1$ y c2 son el puesto en la cola del mensaje en el nodo 1 y 2 respectivamente; Ti es el tiempo de latencia de la tarjeta de comunicación que en la mayoría de los casos esta aproximadamente en $7 \mu \mathrm{s}$. El peor escenario para un mensaje se obtiene al suponer el tamaño máximo de la trama.

$$
T_{m}=(123 \mu s \times 2+7 \mu s)+(123 \mu s \times 2+7 \mu s)=560 \mu s
$$

La latencia de la red a través de N nodos está dada por la ecuación 4 (RuggedCom Inc. I 30 Whitmore Road, 2008):

$$
T_{m}=\sum_{i=1}^{N}\left(T_{P} \times c_{i}+T_{1}\right)=N \times T_{1}+T_{P} \times \sum_{i=1}^{N} c_{i}
$$

Asumiendo un anillo de 50 dispositivos con una falla en el anillo y con la transmisión de un mensaje de punta a punta es decir a través de todos los dispositivos, el mensaje tendría una latencia aproximada de 12,527ms

$$
\begin{aligned}
& T_{m}=50 \times 7 \mu \mathrm{s}+123 \mu \mathrm{s} \times \sum_{\mathrm{i}=1}^{\mathrm{N}} \mathrm{c}_{\mathrm{i}} \\
& T_{\mathrm{m}}=12527 \mu \mathrm{s}=12,527 \mathrm{~ms}
\end{aligned}
$$

Con 50 nodos, el tiempo de desviación en condiciones normales puede exceder los $6 \mathrm{~ms}$. Los valores teóricos de latencia máxima fueron calculados con el peor escenario que se puede presentar en la red y bajo condiciones de carga extrema, sin embargo, este escenario en caso de ocurrir es transitorio y no afecta el desempeño de la red. A partir de estos resultados obtenidos en la red hibrida los dispositivos que conforman el anillo HSR no pueden exceder los 15 IED, con lo que se garantiza tiempos de latencia menores a $4 \mathrm{~ms}$.

\section{RESULTADOS}

El análisis del comportamiento del sistema de comunicación redundante se realizó con ayuda de pruebas controladas de robustez. El sistema de comunicaciones bajo prueba estaba integrado por un total de 70 dispositivos. Entre los equipos que integraron el sistema de comunicación se encuentran cuatro anillos HSR de IED's entre los que están controladores de bahía y relés de protección principal y respaldo; así como dos Switch, un Router y ocho RedBox. Entre los equipos que hacen parte del sistema de automatización se tienen un GPS, dos Gateway, un IHM, dos unidades centrales de registrador de fallas y dos protecciones diferenciales de barras; todos estos equipos conectados a la red PRP a través de RedBox. Como primera fase se interpretó las tramas asociadas a la comunicación entre IED's y Gateway de la subestación, con lo cual se verifico el funcionamiento de acuerdo a la norma IEC 62439-3 a nivel de redundancia de comunicación y se garantizó que el sistema operaba correctamente. Para capturar las tramas en el punto de red asociado al Gateway de la subestación se utilizó el software Wireshark 1.12.6, encontrando un comportamiento estable en las tramas como se presenta en las Figuras 8 y 9. 


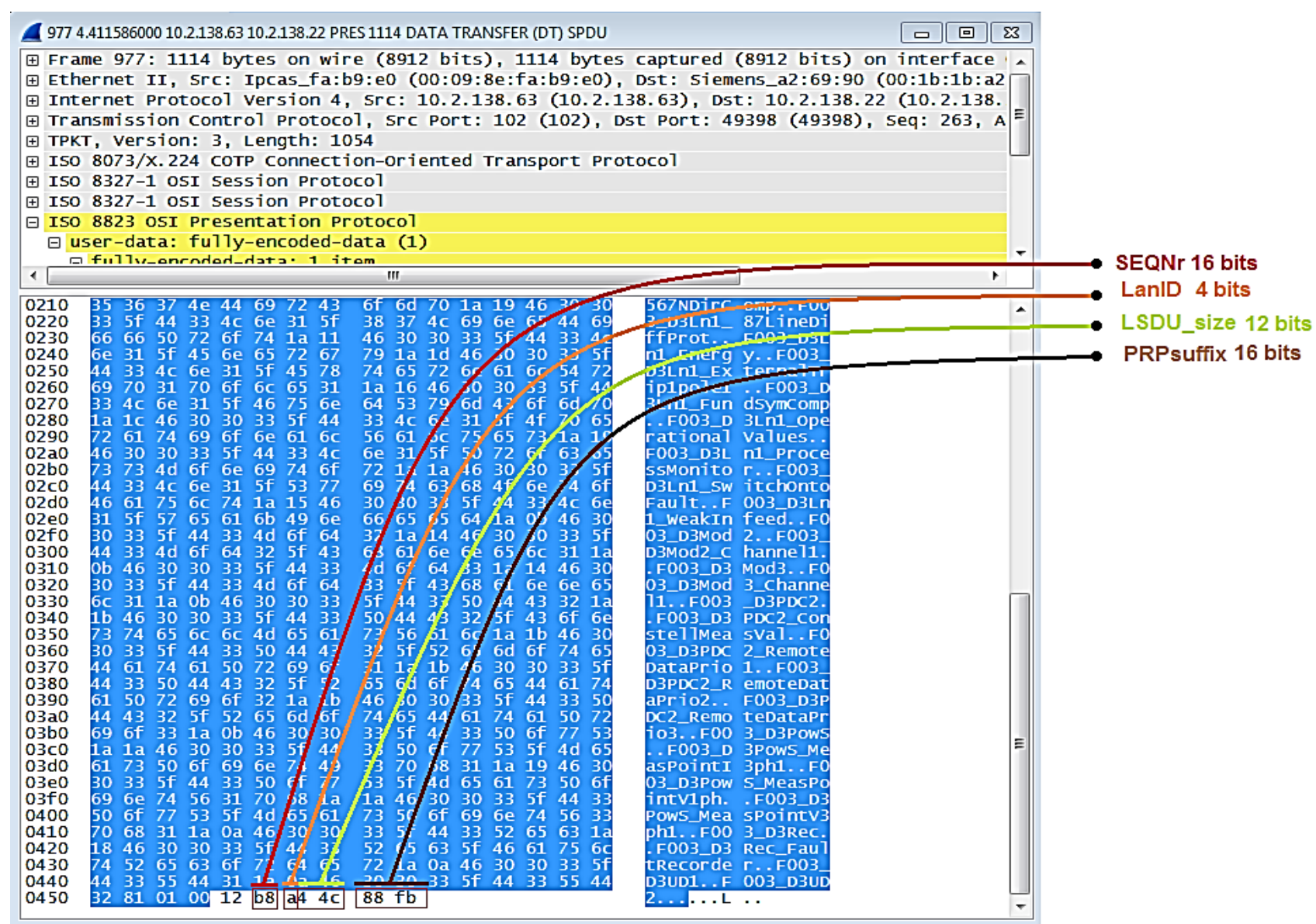

Fig. 8: Trama IEC 61850 entre IED y Gateway con redundancia PRP.

Para el caso presentado en la Figura 8 se tienen que es una trama IEC 61850 mensajería MMS la cual muestra el siguiente comportamiento: i) SEQNr: $b 8=$ En el momento del trace esta trama tenía número de secuencia de 139; ii) LanID: $a$ = Corresponde a 1010 es decir que la trama fue capturada en la red PRP LAN A; iii) LSDU_size: $44 c=$ Corresponde a 010001001100 es decir que la trama original tienen un tamaño de 1100 bytes; y iv) PRPsuffix: $88 \mathrm{fb}=$ Que corresponde al sufijo que identifica a la red PRP.

Otra trama que es importante dentro de la red de comunicaciones es la mensajería GOOSE por lo cual también se realizó la respectiva interpretación para verificar su funcionamiento en la red como se presenta en la Figura 9: i) SEQNr: a0 = En el momento del trace esta trama tenía número de secuencia de 160; ii) LanID: $b=$ Corresponde a 1011 es decir que la trama fue capturada en la red PRP LAN B; iii) LSDU_size: 0 a5 = Corresponde a 000010100101 es decir que la trama original tienen un tamaño de 165 bytes; y v) PRPsuffix: $88 \mathrm{fb}=$ Que corresponde al sufijo que identifica a la red PRP.

Para la prueba de robustez se realizó una avalancha de 100 señales cableadas al sistema de control y protección, simultáneamente ejecutando ping a todos los equipos de la red con diferencias de $4 \mathrm{~ms}$ entre cada solicitud, la prueba se realizó durante 15 minutos en la cual se generaron 6 fallas aleatorias en diferentes puntos de la red dando como resultado los datos presentados en la Figura 10. Durante la prueba no ocurrieron perdidas de paquetes y el tiempo promedio de respuesta entre todos los equipos participantes de la red fue de 2,24ms, lo que presenta un comportamiento aceptable considerando que se simuló una condición de carga en la red y se garantizó los requerimientos de tiempos asociados a los servicios de la norma IEC 61850 durante la prueba. Para la Figura 10, se tiene: Total de paquetes enviados 6524420, total de paquetes recibidos 6524420 , total de perdidas 0 .

Para verificar el comportamiento ante sobre carga de la red se realizó la misma prueba anterior con una duración de 5 minutos, bajo condiciones continuas, con tiempos de $1 \mathrm{~ms}$ entre cada solicitud ping. Durante el evento se perdieron en total 6008 paquetes y el tiempo de recuperación estuvo entre 5 a 12 ms en los 3 equipos que presentaron fallas. Dos de ellos presentaron pérdida de 3003 paquetes y uno de 2 paquetes, el tiempo promedio de respuesta entre todos los equipos participantes de la red fue de $2,30 \mathrm{~ms}$. Se generaron eventos en la subestación simultáneamente a la avalancha sin pérdida de ninguna información obteniendo resoluciones menores a $3 \mathrm{~ms}$. Es de acotar que este tipo de evento por lo general ocurre de manera transitoria 
y de acuerdo con los resultados arrojados por la prueba, presentados en la Figura 11, no se produjo perdida de comunicación en ningún dispositivo participante de la red. Por ende, ante una sobrecarga de las tarjetas puede verse afectada la latencia y posiblemente dependiendo de lo robusta que sea la interface Ethernet de los dispositivos la comunicación en el sistema. Para la figura 11, se tiene: Total de paquetes enviados 2134860 , total de paquetes recibidos 2128852, total de perdidas 6008 .

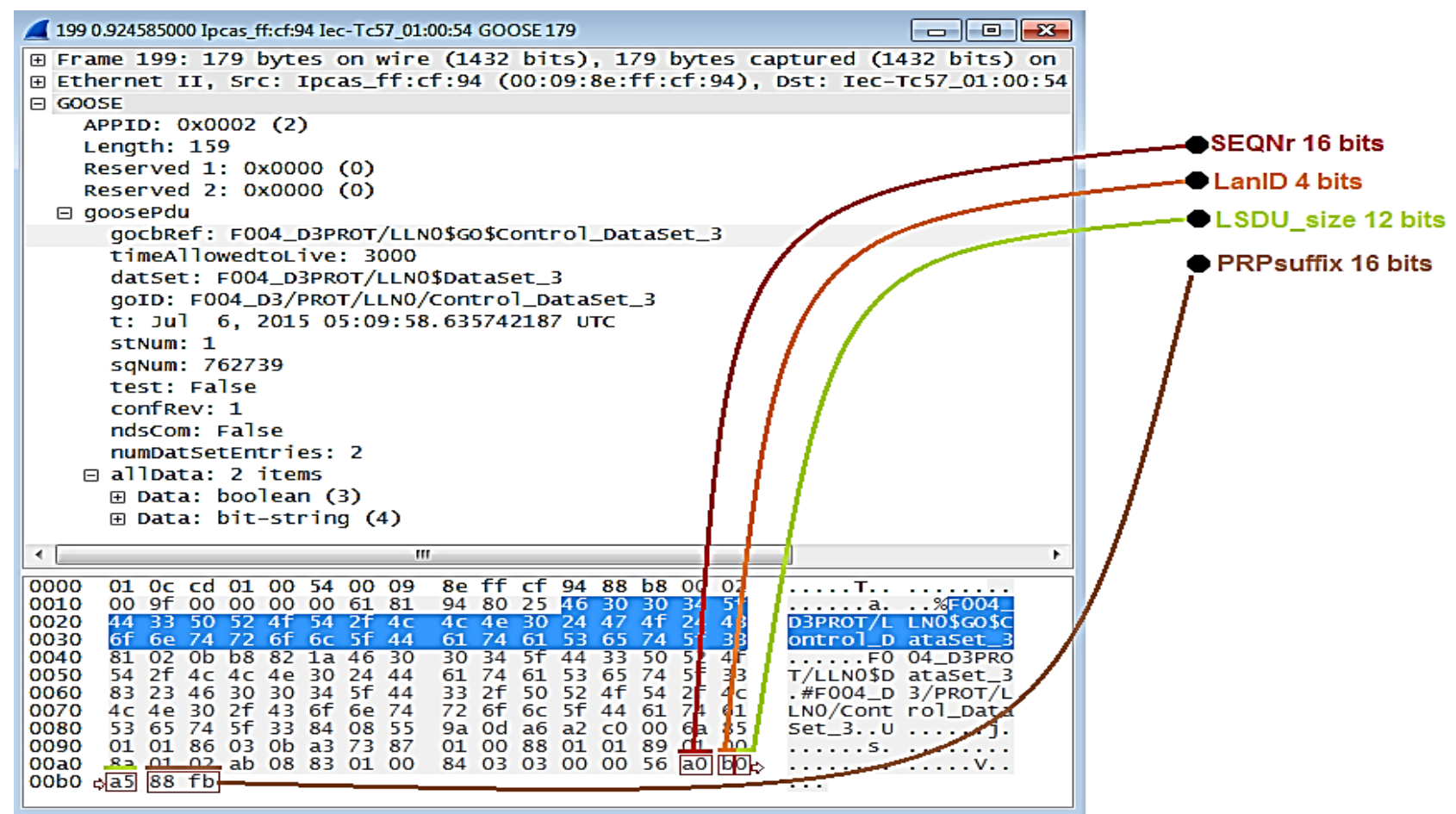

Fig. 9: Trama IEC 61850 GOOSE con redundancia PRP.
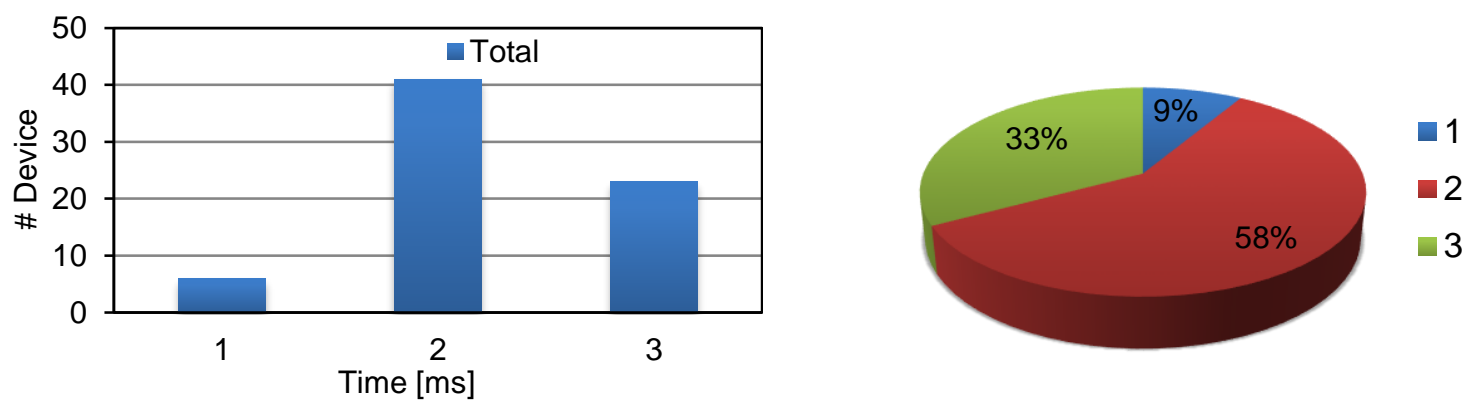

Fig. 10: Comportamiento ante avalancha con ping cada $4 \mathrm{~ms}$ y fallas aleatorias en la Red.

Con el fin de validar los datos obtenidos, se encontraron algunos casos del estudio como el presentado en (Shantanu. K., 2017), en el cual se analizan específicamente los mensajes de alta prioridad transmitidos hacia los extremos remotos de las líneas de transmisión de energía, en el que típicamente se requiere un tiempo de transmisión del dato en el orden de los $10 \mathrm{~ms}$.

En este trabajo se realizaron pruebas con varios escenarios, transfiriendo datos con el protocolo IEC 61850-9-2 Sampled Value (SV) entre dos subestaciones; utilizando redes PRP y vinculando una red con solo dos relés de protección. El laboratorio se llevó a cabo conectando la red LAN A a la tecnología inalámbrica de radio y LAN B conectada a través de una conexión Ethernet, donde encontraron latencias que oscilaron entre los 12-32 ms. Estos valores se consideran altos, comparados con los obtenidos en nuestro estudio, pero que se justifican con el hecho de que nuestros mensajes no atraviesan redes WAN como en el caso de (Shantanu. K., 2017), donde se agregan latencias adicionales a las de la red LAN. 

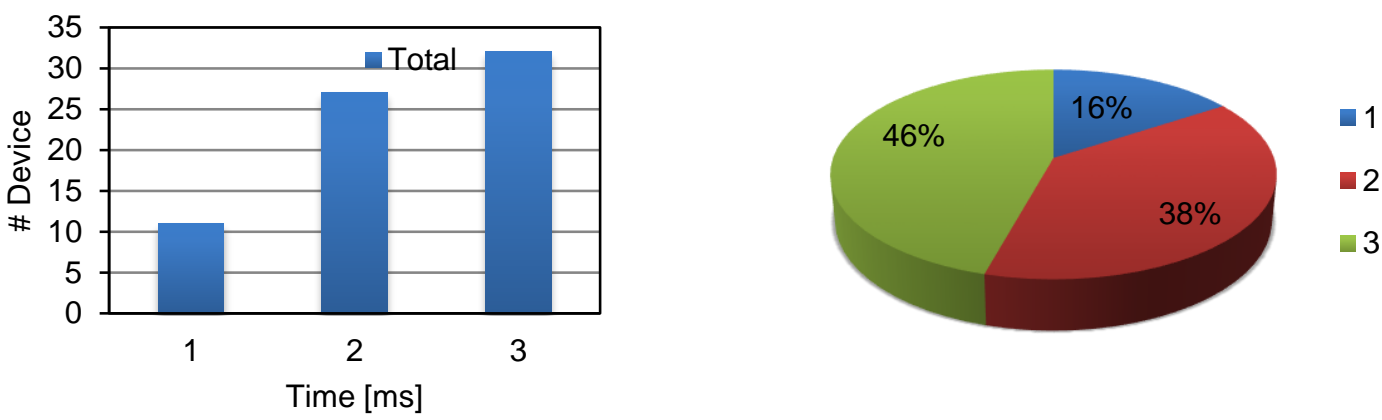

Fig. 11: Comportamiento ante avalancha con ping cada $1 \mathrm{~ms}$ y fallas aleatorias en la Red.

Otra de las investigaciones encontradas es (Gutiérrez, 2017), en donde se emplea una red HSR y se utiliza la tecnología White Rabbit (WR) basada en Ethernet, para la sincronización de tiempo de los dispositivos de red con precisión de nanosegundos. Este esquema está basado en hardware y software abierto y se realizó un laboratorio de pruebas con un anillo HSR de 6 dispositivos, en el que se analizaron tres métricas: latencia, ancho de banda y pérdida de paquetes. La medición de latencia se realizó comparando la marca de tiempo de transmisión y recepción en todo el anillo HSR. El ancho de banda se determinó intercambiando ráfagas de tramas UDP entre dos equipos. La pérdida de paquetes se midió de dos maneras: la primera, con el análisis de las pruebas de ancho de banda y la segunda con las fallas de enlaces dentro del anillo HSR, donde se verificaban que durante las fallas no se perdieran paquetes.

Los investigadores encontraron que los tiempos máximos de latencia en la arquitectura propuesta eran de $7,59 \mu \mathrm{s}$, realizando pruebas con tamaños de paquetes hasta de 372 bytes y con diferentes anchos de banda, obteniendo el caso más crítico con $700 \mathrm{Mbps}$, en donde se presentaron perdidas de paquetes del $2.56 \%$. Comparando los resultados, con un escenario de análisis para $100 \mathrm{Mbps}$, con paquetes de 288 bytes, se concluyó que no se presentaron perdidas de paquetes, concordando con el esquema propuesto en este artículo. Para el escenario de carga extrema de la red para el esquema propuesto, los resultados muestran pérdidas de paquetes del $0.29 \%$, que, si bien no son las mismas condiciones de prueba, se evidencia que ante excesiva carga y saturación de la red puede presentarse pérdidas de paquetes.

Por último, una investigación realizada en (Shantanu. K.,2014), la cual realiza un análisis de una arquitectura conectada en estrella, emulada por software para una subestación de 132/22 kV, con un ancho de banda de $100 \mathrm{Mbps}$ y 39 dispositivos en la red. En esta prueba se analizaron las tramas de mensajería GOOSE, en donde los retardos presentados en la simulación estuvieron entre los $0.4 \mathrm{~ms}$ hasta un máximo de $32 \mathrm{~ms}$, contemplando diferentes escenarios de simulación. En el caso de la arquitectura propuesta en este artículo, se encontró para la transmisión de mensajería GOOSE tiempo promedio del orden de los 3 ms, esto particularmente dado diseño de arquitectura desarrollado en este trabajo.

\section{CONCLUSIONES}

Con la implementación de protocolos de comunicación redundantes HSR y PRP, se logra tener un mayor nivel de disponibilidad y confiabilidad en la automatización y protección de los sistemas de potencia eléctrica. Para garantizar la latencia requerida por los sistemas de control y protección de sistemas eléctricos se recomienda que en un anillo HSR no existan más de 40 dispositivos conectados. Si dentro del sistema eléctrico se implementa funciones de protección a través de GOOSE se recomienda que los anillos HSR no excedan los 15 IED, con lo que se garantiza tiempos de latencia menores a $4 \mathrm{~ms}$. De acuerdo con las pruebas realizadas, se valida que los tiempos de respuesta ante una avalancha y saturación en la red cumplen con los requerimientos necesarios para sistemas eléctricos, ya que en promedio están alrededor de los $3 \mathrm{~ms}$, tiempo permisible para el esquema eléctrico los cuales oscilan desde los 150ms.

Con este trabajo se contribuye a mejorar la aceptación de nuevas tecnologías y en particular la utilización del protocolo IEC61850, comprobando que, teniendo un buen diseño de la red y un protocolo acertado de redundancia a nivel de comunicación, se cumple con todos los requerimientos para el funcionamiento adecuado y seguro del sistema eléctrico. De igual manera, se pretende justificar la implementación de un bus de proceso en una las subestaciones de transmisión de la empresa, además de obtener una reducción en el cableado convencional entre los equipos de potencia y los IED, contribuyendo a los demás agentes del sector con diseños de arquitecturas como guía para soluciones de automatización y protección del sistema eléctrico. 


\section{REFERENCIAS}

Araujo, J.A., J. Lazaro, A. Astarloa, A.Zuloaga y A. Garcia, High availability automation networks: PRP and HSR ring implementations, 2012 IEEE International Symposium on Industrial Electronics, 1197-1202 (2012)

Araujo, J. A., J. Lazaro, A. Astarloa, A.Zuloaga y A. Garcia, A. PRP and HSR version 1 (IEC 62439-3 Ed.2), improvements and a prototype implementation, IECON 2013 - 39 th Annual Conference of the IEEE Industrial Electronics Society, 44104415 (2013)

Goraj, M. y R. Harada, Migration paths for IEC 61850 substation communication networks towards superb redundancy based on hybrid PRP and HSR topologies, $11^{\text {th }}$ IET International Conference on Developments in Power Systems Protection (DPSP 2012), 141-141 (2012)

Gutiérrez-Rivas J.L., J. López-Jiménez, E. Ros y J. Díaz, White Rabbit HSR: a seamless sub-nanosecond redundant timing system with low-latency data capabilities for Smart Grid, IEEE Transactions on Industrial Informatics (2017)

IEC 62439-3 Industrial communication networks - High availability automation networks - Part 3: Parallel Redundancy Protocol (PRP) and High-availability Seamless Redundancy (HSR) (2016)

IEC. Communication networks and systems in substations - Part 7-1: Basic communication structure for substation and feeder equipment - Principles and models (2003)

IEC. IEC 61850-5 Communication networks and systems in substations - Part 5: Communication requirements for functions and device models (2013)

IEC - Communication networks and systems in substations - Part 9-2: Specific Communication Service Mapping (SCSM) - Sampled values over ISO/IEC 8802-3 (2004)

IEC. IEC 61850-8-1 Communication networks and systems in substations Part 8-1: Specific communication service mapping (SCSM) - Mappings to MMS (ISO 9506-1 and ISO 9506-2) and to ISO/IEC 8802-3 (2011)

IEC 61850-7-2 Communication networks and systems in substations (2010)

IEEE 802.1D: 2004 Local and Metropolitan Area Networks: Media Access Control (Mac) Bridges (2004)

IEEE SA - 802.1D-1990 - Standard for Local and Metropolitan Area Networks: Media Access Control (MAC) Bridges (1990) Jiang, X., High Availability Seamless Ring Protocol Implementation in FPGA (2009)

Kirrmann, H., HSR - High-availability Seamless Redundancy Zero-delay recovery fault-tolerance in Industrial Ethernet in a ring or meshed topology (2017)

Kumar, S., N. Das y S. Islam, Performance evaluation of two interconnected high voltage utility substations using PRP topology based on IEC 62439-3, Australasian Universities Power Engineering Conference (AUPEC), 1-5 (2017)

Kumar, S., N. Das y S. Islam, Performance analysis of substation automation systems architecture based on IEC 61850 , Australasian Universities Power Engineering Conference (AUPEC), 1-6 (2014)

Leslie, M., Calculating Latency on a Switched Ethernet Network (2008)

Molina, E., E. Jacob, N. Toledo y A. Astarloa, Performance Enhancement of High-Availability Seamless Redundancy (HSR) Networks Using OpenFlow, IEEE Communications Letters, 20(2), 364-367 (2016)

Shantanu Kumar, S.I., Software Implementation of Two Seamless Redundant Topologies in a Digital Protection System based on IEC 62439-3 (2016)

Standards Committee of the IEEE Computer Society, M. IEEE std 802.3-2005, IEEE Standard for physical layer and data link layer media access control (MAC) of wired Ethernet (2005)

Weibel, H., Tutorial on Parallel Redundancy Protocol (PRP) (2013)

Yu, J.T., Applying IEEE 802.1w (RSTP) to Improve Service Availability (2003)

Zapata, G., J. Cardillo y E. Chacón, Aportes Metodológicos para el Diseño de Sistemas de Supervisión de Procesos Continuos. Información tecnológica, 22(3), 97-114 (2011) 
